\title{
OBTAINING ANALYTIC FUNCTIONS AND CONJUGATE HARMONIC FUNCTIONS*
}

\section{By RAIMOND A. STRUBLE (North Carolina State University)}

E. V. Laitone [2] has called our attention to a useful (apparently not so well advertised) procedure for obtaining an analytic function from one of its conjugate harmonics. The idea is simply this: if $f(z)=f(x+i y)=\phi(x, y)+i \psi(x, y)$ is analytic in a neighborhood of some point $z_{0}=x_{0}+i y_{0}$, then it can be recovered from either of its conjugate harmonics through the integration formulas,

$$
\begin{aligned}
f(z)=\int\left[\frac{\partial \phi}{\partial x}\left(z-i y_{0}, y_{0}\right)-i \frac{\partial \phi}{\partial y}\left(z-i y_{0}, y_{0}\right)\right] d z= & \int\left[\frac{\partial \psi}{\partial y}\left(z-i y_{0}, y_{0}\right)\right. \\
& \left.+i \frac{\partial \psi}{\partial x}\left(z-i y_{0}, y_{0}\right)\right] d z
\end{aligned}
$$

in the "variable" $z$. These formulas are readily obtained from the Cauchy-Riemann equations once it is observed that

$$
f(z)=\phi\left(z-i y_{0}, y_{0}\right)+i \psi\left(z-i y_{0}, y_{0}\right)
$$

holds for all $z$ in some neighborhood of $z_{0}$.

Actually, Laitone derived (2) only for $y_{0}=0$, using the conjugate complex function $\bar{f}(\bar{z})$. An alternative (and perhaps more direct and illuminating) derivation can be given using Taylor series. For if

$$
\phi\left(u+x_{0}, y_{0}\right)=\sum a_{n} u^{n}, \psi\left(u+x_{0}, y_{0}\right)=\sum b_{n} u^{n}
$$

are the Taylor series expansions (in the first variable) of $\phi$ and $\psi$ about $x_{0}$, then

$$
a_{n}=\frac{1}{n !} \frac{\partial^{n} \phi}{\partial x^{n}}\left(x_{0}, y_{0}\right), b_{n}=\frac{1}{n !} \frac{\partial^{n} \psi}{\partial x^{n}}\left(x_{0}, y_{0}\right)
$$

hold for all $n$. But $d^{n} f / d z^{n}=\partial^{n} \phi / \partial x^{n}+i \partial^{n} \psi / \partial x^{n}$, so that the Taylor series expansion of $f$ about $z_{0}=x_{0}+i y_{0}$ gives

$$
\begin{aligned}
f(z)=\sum \frac{1}{n !} \frac{d^{n} f}{d z^{n}}\left(z_{0}\right)\left[z-z_{0}\right]^{n}=\sum\left(a_{n}+i b_{n}\right)\left[z-\left(x_{0}+i y_{0}\right)\right]^{n}= \\
\phi\left(z-i y_{0}, y_{0}\right)+i \psi\left(z-i y_{0}, y_{0}\right)
\end{aligned}
$$

where we have used (3) with $u=z-\left(x_{0}+i y_{0}\right)$.

Using analogous Taylor series and $d^{n} f / d z^{n}=(-i)^{n} \partial^{n} \phi / \partial y^{n}+(-i)^{n-1} \partial^{n} \psi / \partial y^{n}$, we similarly obtain an analogous identity

$$
f(z)=\phi\left(x_{0},-i z+i x_{0}\right)+i \psi\left(x_{0},-i z+i x_{0}\right) .
$$

\footnotetext{
${ }^{*}$ Received December 19, 1977; revised version received June 29, 1978.
} 
This alternative derivation might help to explain some of the symbolism used in (1) (the permanence of the Cauchy-Riemann equations under analytic continuation etc.) and so help (along with Laitone's examples) to rescue the idea from near oblivion.

Even more helpful is the Taylor series (in two variables)

$$
\phi\left(h+x_{0}, k+y_{0}\right)=\sum \frac{1}{n !}\left(h \frac{\partial}{\partial x_{0}}+k \frac{\partial}{\partial y_{0}}\right)^{n} \phi\left(x_{0}, y_{0}\right) .
$$

For with $h=u / 2$ and $k=-i u / 2$, we obtain the identity

$$
2 \phi\left(\frac{u}{2}+x_{0}, \frac{-i u}{2}+y_{0}\right)=\sum \frac{2}{n !}\left(\frac{u}{2}\right)^{n}\left(\frac{\partial}{\partial x_{0}}-i \frac{\partial}{\partial y_{0}}\right)^{n} \phi\left(x_{0}, y_{0}\right) .
$$

But from the Cauchy-Riemann equations we have

$$
\left(\partial / \partial x_{0}-i \partial / \partial y_{0}\right) \phi\left(x_{0}, y_{0}\right)=\partial \phi\left(x_{0}, y_{0}\right) / \partial x_{0}+i \partial \psi\left(x_{0}, y_{0}\right) / \partial x_{0}=d f\left(z_{0}\right) / d z_{0} .
$$

Hence $\left(\partial / \partial x_{0}-i \partial / \partial y_{0}\right)^{2} \phi\left(x_{0}, y_{0}\right)=\left(\partial / \partial x_{0}-i \partial / \partial y_{0}\right) d f\left(z_{0}\right) / d z_{0}=2 d^{2} f\left(z_{0}\right) / d z_{0}^{2}$, and so by induction, $\left(\partial / \partial x_{0}-i \partial / \partial y_{0}\right)^{n} \phi\left(x_{0}, y_{0}\right)=2^{n-1} d^{n} f\left(z_{0}\right) / d z_{0}^{n}$ holds for all $n \geq 1$. The series in $(5)$ then becomes

$$
\phi\left(x_{0}, y_{0}\right)-i \psi\left(x_{0}, y_{0}\right)+\sum \frac{1}{n !}\left(d^{n} f\left(z_{0}\right) / d z_{0}^{n}\right) u^{n}
$$

and with $u=z-z_{0}$ we obtain what may be the simplest of all recovery formulas,

$$
\begin{aligned}
f(z) & =2 \phi\left(\frac{z-z_{0}}{2}+x_{0}, \frac{-i\left(z-z_{0}\right)}{2}+y_{0}\right)-\phi\left(x_{0}, y_{0}\right)+i \psi\left(x_{0}, y_{0}\right) . \\
( & \left.=2 i \psi\left(\frac{z-z_{0}}{2}+x_{0}, \frac{-i\left(z-z_{0}\right)}{2}+y_{0}\right)-i \psi\left(x_{0}, y_{0}\right)+\phi\left(x_{0}, y_{0}\right)\right)
\end{aligned}
$$

The simplicity in (6), of course, lies in the fact that no integrations are required to obtain $f$ from $\phi($ or $\psi)$. Once (6) is anticipated, it can be verified directly. For the left-hand and right-hand members are clearly equal when $z=z_{0}$, and upon using the Cauchy-Riemann equations, their derivatives can be seen to be equal. In fact, $\left(z-z_{0}\right) / 2+x_{0}=\left(z+\bar{z}_{0}\right) / 2$, $i\left(z-z_{0}\right) / 2+y_{0}=\left(z-\bar{z}_{0}\right) / 2 i$, and

$$
\begin{aligned}
\frac{d}{d z} 2 \phi\left(\frac{z+\bar{z}_{0}}{2}, \frac{z-\bar{z}_{0}}{2 i}\right) & =\frac{\partial \phi}{\partial x}\left(\frac{z+\bar{z}_{0}}{2}, \frac{z-\bar{z}_{0}}{2 i}\right)-i \frac{\partial \phi}{\partial y}\left(\frac{z+\bar{z}_{0}}{2}, \frac{z-\bar{z}_{0}}{2 i}\right) \\
& =\frac{\partial \phi}{\partial x}\left(\frac{z+\bar{z}_{0}}{2}, \frac{z-\bar{z}_{0}}{2 i}\right)+i \frac{\partial \psi}{\partial x}\left(\frac{z+\bar{z}_{0}}{2}, \frac{z-\bar{z}_{0}}{2 i}\right) \\
& =\frac{d f}{d z}(X+i Y) \quad \text { with } \quad X=\frac{z+\bar{z}_{0}}{2}, Y=\frac{z-\bar{z}_{0}}{2 i} \\
& =\frac{d f}{d z}(z) .
\end{aligned}
$$

The circumstance that $X+i Y$ becomes simply $z$ when $X=\left(z+\bar{z}_{0}\right) / 2$ and $Y=\left(z-\bar{z}_{0}\right) / 2 i$ plays a crucial role here, of course. Similar verifications of (2) and of (4) can be made.

Eq. (6), for $z_{0}=0$, has been derived (somewhat heuristically) in [1] and [3] and shows that a real analytic function $\phi$ of two variables is harmonic, say near the origin, iff

$$
\phi(x, y)=2 \operatorname{Re} \phi\left(\frac{x+i y}{2}, \frac{y-i x}{2}\right)-\phi(0,0)
$$


holds there. Of considerable interest also is the companion equation giving the conjugate harmonic function,

$$
\psi(x, y)=2 \operatorname{Im} \phi\left(\frac{x+i y}{2}, \frac{y-i x}{2}\right)+\psi(0,0) .
$$

Thus each stream function $\psi$ can be expressed algebraically (without differentiations or integrations) in terms of the corresponding potential function $\phi$, and vice versa.

Perhaps the identities (2), (4), (6) and (8) (along with these elementary derivations) should be better known than they appear to be.

\section{REFERENCES}

[1] L. V. Ahlfors, Complex analysis, McGraw-Hill Book Company, New York, 1966, p. 27

[2] E. V. Laitone, Relation of the conjugate harmonic functions to f(z), A mer. Math. Monthly 84, 281-283 (1977)

[3] L. M. Milne-Thomson, Theoretical hydrodynamics, The Macmillan Company, New York, 4th ed., 1962, 5th ed., 1967 